\title{
PHENOTYPIC SELECTION IN AUTOPOLYPLOIDS
}

\author{
A. J. WRIGHT \\ Plant Breeding Institute, Cambridge CB2 $2 L Q$
}

Received 8.v.75

\section{SUMMARY}

\begin{abstract}
A simple demonstration, applicable to any ploidy level, that response to selection measured after one generation of random mating is proportional to the gametic variance, is given. The reduction in the coefficients of variance components during subsequent random mating generations is related to the approach of the population structure to various types of equilibria.
\end{abstract}

\section{INTRODUCTION}

THE behaviour of a diploid population under random mating after selection was first described by Griffing (1960). He showed that while the mean of the selected population is initially expected to exceed that of the unselected equilibrium population by $\left(i / \sigma_{x}\right)\left(\sigma_{A}^{2}+\frac{1}{2} \sigma_{A A}^{2}\right)$, the part of this advantage due to additive $\times$ additive epistasis is lost during subsequent random mating generations. The reason for this change is the return of the population, at a rate depending on the linkage relations of the loci involved, to the linkage equilibrium which was destroyed by selection.

Recently Hill and Haag (1974) have postulated that the digenic component of variance will be involved in a similar change in mean of selected autotetraploid populations during random mating. Other workers (Morley and Heinrichs, 1960; Levings and Dudley, 1963) have based tetraploid response formulae on the parent-offspring covariance, a statistic which includes a digenic component. In fact it is intuitively reasonable to expect that for an organism at any ploidy level response measured at equilibrium will depend only on the genic or additive component of variance, whereas immediate response will be proportional to the covariance of offspring and parent.

The purpose of this paper is to give a simple derivation of response formulae which can be applied to any level of ploidy and which allows an explicit demonstration of the above hypothesis.

\section{THEORY}

The simplicity of this derivation rests on a useful property of the initial population when this is assumed to be in equilibrium and without double reduction. The gametic output of an equilibrium zygotic generation is identical to the gametic array which formed it, and it can be further shown that the array of gametes uniting to form all individuals of a specific genotype is identical to that which is subsequently released by these individuals. This situation can be treated as if each individual gives rise in equal proportions to the two gametic types from which it was formed, allowing considerable simplification of the notation and procedures involved.

The genotypic model initially involves gametic effects which can later be interpreted in terms of genic effects and interactions. There are $n$ 
gametic types uniting at random to form the initial population. Thus, the population mean is

$$
\bar{y}=\Sigma_{i}^{n} \Sigma_{j}^{n} f_{i} f_{j} y_{i j}
$$

where $f_{i}$ and $f_{j}$ are the frequencies of the $i$ th and $j$ th gametes, and $y_{i j}$ is the value of their zygote. Also:

$$
y_{i j}=y+y_{i}^{\prime}+y_{j}^{\prime}+i_{i j}^{\prime}, \text { and } y_{i j}^{\prime}=y_{i j}-\bar{y} \text { etc. }
$$

where $y_{i}^{\prime}$ is the average effect of the $i$ th gamete and $i_{i j}^{\prime}$ its interaction with the $j$ th. These effects are defined such that

$$
\Sigma_{i}^{n} f_{i} y_{i}^{\prime}=\Sigma_{j}^{n} f_{j} y_{j}^{\prime}=\Sigma_{i}^{n} f_{i} i_{i j}^{\prime}=\Sigma_{j}^{n} f_{j} i_{i j}^{\prime}=0 .
$$

Assuming small changes of gametic frequency under selection, the change of population mean can be expressed as:

Now

$$
\Delta \bar{y}=\Sigma_{k}^{n}\left(\Delta f_{k} \cdot \frac{d \bar{y}}{d f_{k}}\right) k
$$

$$
\Delta f_{k}=\Delta_{x} \frac{d f_{k}}{d x}=\left(i \sigma_{x}\right) b\left(f_{k} / x\right)=\frac{i}{\sigma_{x}} \operatorname{cov}\left(f_{k} \cdot x\right)=\frac{i}{\sigma_{x}} \Sigma_{i}^{n} \Sigma_{j}^{n} f_{i} f_{j} y_{i j}^{\prime} t_{k(i j)},
$$

where the $x$ are the phenotypic measurements on which selection is based, and are equal to the $y_{i j}$ plus uncorrelated environmental deviations. Also $i$ is the selection intensity, and $t_{k(i j)}$ the proportion of gametes of type $k$ produced by genotype $i j$. From the property of the equilibrium population discussed above, $t_{k(i j)}=\frac{1}{2}$ when $k=i$ or $j$, and 0 otherwise. Thus:

$$
\Delta f_{k}=\frac{i}{\sigma_{x}}\left(\frac{1}{2} f_{k} \Sigma_{j}^{n} f_{j} y_{k j}^{\prime}+\frac{1}{2} f_{k} \Sigma_{i}^{n} f_{i} y_{i k}^{\prime}\right)=\frac{i}{\sigma_{x}} f_{k} y_{k}^{\prime} .
$$

The value of $d \bar{y} / d f_{k}$ is found most easily by differentiation:

$$
\bar{y}=\Sigma_{i}^{n} \Sigma_{j}^{n} f_{i} f_{j} y_{i j}=\mu+\Sigma_{i}^{n} f_{i} \Sigma_{j}^{n} f_{j} y_{i j}^{\prime},
$$

where $\mu$ is treated as constant. Therefore,

Using (1):

$$
\frac{d \bar{y}}{d f_{k}}=\Sigma_{j}^{n} f_{j} y_{k j}^{\prime}+\Sigma_{i}^{n} f_{i} y_{i k}^{\prime}=2 y_{k}^{\prime} .
$$

$$
\Delta \bar{y}=\frac{i}{\sigma_{x}} \cdot 2 \cdot\left(\Sigma_{k}^{n} f_{k} y_{k}^{\prime 2}\right)
$$

The term in brackets here is clearly the population variance of average gametic effects or the gametic variance (Crow and Kimura, 1970), and is therefore equal to the covariance of two individuals with one gamete in common, i.e. parent and offspring. Methods for deriving the expectation of parent-offspring and other covariances for any level of ploidy are given by Kempthorne (1957). However, these can be obtained directly using his model for genic effects and interactions. Essentially, this takes the form:

$$
\text { genotype }=\mu+\Sigma_{i} \alpha_{i}+\Sigma_{j} d_{j}+\Sigma_{l} t_{l}+\ldots+\Sigma_{m}(\alpha \alpha)_{m}+\ldots e t c .,
$$

where $\alpha_{i}, d_{j}$ and $t_{l}$ are mono-, di- and tri-genic intra-locus effects, and summation is over all single alleles, all pairs, all triplets, etc. For a given 
level of ploidy $(n)$ the number of terms in any of these summations involving intra-locus terms is:

$$
{ }^{n} C_{r}=n(n-1) \ldots(n-r+1) / r !,
$$

where $r$ is the order of interaction of alleles (i.e. $r=2$ for digenic terms). A gamete has one-half of the ploidy of the zygote and so contains only ${ }^{\left({ }^{(} n\right)} C_{r}$ of the terms. Since the distributions of all effects throughout the population are independent, the proportion of variance of any type of effect accounted for by the gametic variance is

$$
\frac{{ }^{\left(\frac{1}{2} n\right)} C_{r}}{{ }^{n} C_{r}}=\frac{\frac{1}{2} n\left(\frac{1}{2} n-1\right) \ldots\left(\frac{1}{2} n-r+1\right)}{n(n-1) \ldots(n-r+1)} .
$$

By similar reasoning, the coefficients for effects arising from two locus epistatic terms $\left(\alpha \alpha_{m}\right.$ etc.) are found to be the products of those for the appropriate single locus effects, as is the case for diploids (Kempthorne, 1957). The resultant expectations of gametic variance and hence of one-half the numerators for response formulae for the diploid, tetraploid and hexaploid cases are given in table 1 , and are in agreement with expectations of parentoffspring covariances given elsewhere (Kempthorne, 1957; Levings and Dudley, 1963).

TABLE 1

\begin{tabular}{|c|c|c|c|c|c|c|c|c|c|}
\hline & \multicolumn{9}{|c|}{ Variance components } \\
\hline & $\alpha$ & $d$ & $t$ & $(\alpha \alpha)$ & $(\alpha d)$ & $(\alpha t)$ & $(d d)$ & $(d t)$ & $(t t)$ \\
\hline $\begin{array}{l}\text { Diploid } \\
\text { Tetraploid } \\
\text { Hexaploid }\end{array}$ & $\begin{array}{l}\frac{1}{2} \\
\frac{1}{2} \\
\frac{1}{2}\end{array}$ & $\begin{array}{l}0 \\
\frac{1}{6} \\
\frac{1}{5}\end{array}$ & $\begin{array}{r}0 \\
0 \\
\frac{1}{20}\end{array}$ & $\begin{array}{l}0 \\
\frac{1}{4} \\
\frac{1}{4}\end{array}$ & $\begin{array}{c}0 \\
\frac{1}{12} \\
\frac{1}{10}\end{array}$ & $\begin{array}{r}0 \\
0 \\
\frac{1}{40}\end{array}$ & $\begin{array}{c}0 \\
\frac{1}{36} \\
\frac{1}{25}\end{array}$ & $\begin{array}{r}0 \\
0 \\
\frac{1}{100}\end{array}$ & $\begin{array}{r}0 \\
0 \\
\frac{1}{40} \overline{0}\end{array}$ \\
\hline
\end{tabular}

Gametic variance for various ploidy levels in terms of genic effects and interactions

The gain expected after the population has regained gamete phase equilibrium can be obtained by redefining all gametic terms as genic terms throughout, or by noting that $d \bar{y} / d f_{k}$ is the sum of derivatives of $\vec{y}$ with respect to allele frequency for all alleles carried by the gamete, and that at equilibrium these derivatives are functions only of allelic effects. Thus, response depends on the covariance of gametic and allelic effects, which, because of the random association of alleles into gametes, is equal to the allelic or additive variance.

\section{Discussion}

The demonstration that immediate selection response is determined by gametes and equilibrium response by genes is a simple extension of Griffing's (1960) work for the diploid case. The reduction of the numerator of response formulae during random mating after selection in fact takes place in several stages each relating to the attainment of a different type of equilibrium by the population. For polyploids, four such equilibria are shown in table 2, and in each case the variance contributing to response is the variance among units of inheritance which have reached equilibrium. The 
loss of the environmental component of variance may appear to be a trivial case, but can nevertheless be included as part of this general phenomenon. The final two types of equilibria are not well distinguished in polyploids

TABLE 2

Types of equilibria and associated variance components

Type of equilibrium

1. Phenotype = Genotype

2. Zygote array $=$ square of gamete array

3. Zygote array at any locus $=$ square of gene array (HardyWeinberg)

4. Zygote array $=$ square of gene array (linkage)
Variance contributing to response

Occurrence General

Tetraploids

Removal of plants

Genotypic

$$
\begin{aligned}
V_{A} & +V_{D}+V_{T}+V_{F}+V_{A A} \\
& +V_{A D}+V_{A T}+V_{A F} \\
& +V_{D D}+V_{D T}+V_{D F} \\
& +V_{T T}+V_{T F}+V_{F F}
\end{aligned}
$$

One random mating

generation

$2 \times$ Gametic

$V_{A}+\frac{1}{3} V_{D}+\frac{1}{2} V_{A A}+\frac{1}{6} V_{A D}$
$\quad+\frac{1}{18} V_{D D}$

$n$ random mating

generations

$-\quad V_{A}+\frac{1}{2} V_{A A}$

$2 \times$ Genic $\quad V_{A}$

$n$ random mating

generations

since they are approached concurrently through several generations, and are consequently often treated together (Bennett, 1954). Equilibrium 3 is therefore never achieved without some accompanying decrease in epistatic components, and so the variance given in table 2 is hypothetical only.

The rate of reduction in the coefficients of variance from intralocus sources, and of those for epistasis from unlinked loci, is given simply by applying a recurrence relation to the coefficients after one generation. Hence for tetraploids, the variance still contributing to response after $m$ generations of random mating is

$$
V_{A}+\left(\frac{1}{3}\right)^{m} V_{D}+\left(\frac{1}{2}\right)^{m} V_{A A}+\left(\frac{1}{6}\right)^{m} V_{A D}+\left(\frac{1}{18}\right)^{m} V_{D D}
$$

The coefficients for linked epistatic genes are not known but must be greater than those for unlinked genes. The change of population mean is therefore dependent on the relative and absolute magnitudes of several variance components and linkage parameters, and is likely to be an uneven, unpredictable process.

\section{REFERENCES}

BennetT, J. H. 1954. Panmixia with tetrasomic and hexasomic inheritance. Genetics, 39, 150-158.

Grow, J. F., AND kimura, M. 1970. An Introduction to Population Genetics Theory. Harper \& Row, London.

GRIFFING, B. 1960. Theoretical consequences of truncation selection based on the individual phenotype. Aust. F. Biol. Sci., 13, 307-343. 
HILL, R. R., AND HAAG, W. L. 1974. Comparison of selection methods for autotetraploids. I. Theoretical. Crop Sci., 14, 587-590.

kEMPthorne, o. 1957. An Introduction to Genetic Statistics. Wiley, New York.

LEVINGS, C. S., AND DUDLEY, J. W. 1963. Evaluation of certain mating designs for estimation of genetic variance in autotetraploid alfalfa. Crop Sci., 3, 532-535.

MORLEY, F. H. W., AND HEINRIGHS, D. H. 1960. Breeding for creeping root in alfalfa. Can. 7. Pl. Sci., 40, 424-433. 